\title{
SSEA-3 as a novel amplifying cancer cell surface marker in colorectal cancers
}

\author{
YOZO SUZUKI ${ }^{1}$, NAOTSUGU HARAGUCHI ${ }^{1}$, HIDEKAZU TAKAHASHI $^{1}$, MAMORU UEMURA ${ }^{1}$, \\ JUNICHI NISHIMURA ${ }^{1}$, TAISHI HATA ${ }^{1}$, ICHIRO TAKEMASA ${ }^{1}$, TSUNEKAZU MIZUSHIMA ${ }^{1}$, \\ HIDESHI ISHII $^{2}$, YUICHIRO DOKI ${ }^{1}$, MASAKI MORI ${ }^{1}$ and HIROFUMI YAMAMOTO ${ }^{1}$ \\ Departments of ${ }^{1}$ Gastroenterological Surgery and ${ }^{2}$ Frontier Science for Cancer and Chemotherapy, \\ Graduate School of Medicine, Osaka University, Suita, Osaka 565-0871, Japan
}

Received August 7, 2012; Accepted November 8, 2012

DOI: $10.3892 /$ ijo.2012.1713

\begin{abstract}
Findings from studies on stem cells have been applied to cancer stem cell (CSC) research, but little is known about the relationship between ES cell-related cell surface markers and CSCs. In this study, we focused on stage-specific embryonic antigen 3 (SSEA-3), a marker of mesenchymal stem cells and Muse cells in colorectal cancer (CRC). Expression of SSEA-3 in human CRC cell lines and clinical specimens, specifically the relationship of SSEA-3 expression and the representative CSC markers (CD44, CD166, ALDH, CD24 and CD26) as well as with mesenchymal stem cell/Muse cell marker (CD105) were assessed. To characterize SSEA-3-expressing cells, tumorigenicity, sphere formation ability, expression of iPS genes (Oct4, NANOG, SOX2 and c-Myc), cell proliferation and cell cycle status were assessed. SSEA-3 expression was identified in Caco-2, DLD-1, HT-29, SW480 and HCT116, but not in CaR-1 cells. No significant relationship between SSEA-3 and other stem cell markers was detected. SSEA-3 ${ }^{+}$ cells showed increased tumorigenicity in vivo, but lower sphere formation ability in vitro than SSEA-3-. iPS gene expression was not correlated with SSEA-3 expression status. SSEA-3 ${ }^{+}$ cells showed higher proliferative ability than SSEA-3- through enhanced cell cycles by decreased expression of $\mathrm{p} 21^{\mathrm{Cip} 1 / \text { Wafl }}$ and $\mathrm{p} 27^{\mathrm{Kip} 1}$. Immunofluorescence analysis in clinical specimens indicated that expression of SSEA-3 is limited to stromal cells in normal mucosa but broad in poorly differentiated adenocarcinoma. These observations indicated that SSEA $-3^{+}$cells in CRC have immature phenotype but decreased self-renewal ability and may function as tumor transient amplifying cells or delayed contributing tumor-initiating cells.
\end{abstract}

Correspondence to: Dr Naotsugu Haraguchi, Department of Gastroenterological Surgery, Graduate School of Medicine, Osaka University, 2-2 Yamadaoka, Suita, Osaka 565-0871, Japan

E-mail: nharaguchi@gesurg.med.osaka-u.ac.jp

Key words: stage-specific embryonic antigen 3, colorectal cancer, cancer stem cell, cell proliferation

\section{Introduction}

Recent vigorous research of somatic stem cells, embryonic stem (ES) cells and induced pluripotent stem (iPS) cells clarified several novel key regulators associated with cellular pluripotency, undifferentiated phenotype, self-renewal and stem cell maintenance. In cancer research, especially in cancer stem cell (CSC) research, some reports focused on the expression of pluripotency-associated factors and indicated their correlation with cancer cell aggressiveness (1), chemoradiotherapy resistance (2) and cancer stem-like cell properties (3), but little is known about the relationship between ES cell-related cell surface markers and cancer. In this study, we focused on stage-specific embryonic antigen 3 (SSEA-3) in colorectal cancer (CRC). SSEAs are globoseries glycolipids and are known to consist of 3 species; SSEA-1, 3 and 4. SSEA-1 and SSEA-4 were established through immunization of animals with human embryonic carcinoma cells, and SSEA-3 with 4- to 8-cell stage mouse embryos (4-6). SSEA-4 is similar to SSEA-3 in terms of structure; they share the same globoseries glycolipid but SSEA-4 has an additional terminal sialic acid moiety (7). SSEA-1 and SSEA-3/-4 are reportedly expressed on the surface of the murine $(8-10)$ and human $(11,12)$ pluripotent stem cells; inner cell mass of early embryos, ES cells and iPS cells, respectively. Though expression of SSEAs are thought to be attenuated during the process of differentiation, a recent report revealed that a small group of SSEA-3 expressing cells; multilineage differentiating stress enduring (Muse) cells, exist in adult skin fibroblasts and bone marrow stroma and that they possess stress tolerance and endogenous pluripotency (13). In cancer, expression of SSEA-1 is related to the poor prognosis (14) and tumor-initiating capacity in human glioblastoma (15), whereas the reduced expression of SSEA-4 is correlated with advanced tumor stages and poor tumor cell differentiation in ovarian cancer (16). In contrast to SSEA-1 and SSEA-4, there are only a few reports that have assessed SSEA-3 expression in cancer; in breast cancer, $77.5 \%$ of clinical breast cancer samples express SSEA-3 with a broad positive ratio (17), and in non-small cell lung cancer, SSEA-3 expression increases after exposure to multiple anti-cancer drugs (2). To the best of our knowledge, there are no reports that have clearly assessed SSEA-3 expression and its biological characteristics in gastro- 
enterological cancer. Furthermore, there are no reports that have assessed SSEA-3 expression in association with CSC. In addition, human colorectal epithelial cells were shown to be SSEA-3 positive (17), implying that CRC tissue also contains SSEA-3 expressing immature subsets.

In this study, we assessed the existence of SSEA $-3^{+}$cells in the cell lines of CRC. Next, we investigated the relationship between SSEA-3 expression and representative colorectal CSC or Muse cell marker, tumorigenic activity and sphere formation activity to assess for CSC-like properties. Then, we assessed the cell proliferation activity of SSEA $-3^{+}$cells to characterize them. Finally, we assessed the distribution of SSEA- $3^{+}$cells to confirm the existence of SSEA- $3^{+}$cells in clinical specimens.

\section{Materials and methods}

Patients and tissue samples. Surgically resected CRC samples were obtained from 10 patients after informed consent from Osaka University Medical Hospital with approval of the research ethics board. All specimens were embedded in Tissue-Tek O.C.T. compound (Sakura Finetek, Tokyo, Japan), rapidly frozen by immersion in liquid nitrogen and stored at $-80^{\circ} \mathrm{C}$.

Cell lines and culture. Human CRC cell line Caco-2, DLD-1, HCT116, HT-29 and SW480 were obtained from American Type Culture Collection (Manassas, VA) and Car-1 from Japanese Collection of Research Bioresources (Osaka, Japan). Caco-2 and Car-1 were maintained in Eagle's minimum essential medium (Wako Pure Chemical Industries, Tokyo, Japan), DLD-1, SW480 and NTERA-2 were maintained in Dulbecco's minimum essential medium (Wako Pure Chemical Industries), and HCT116 and HT-29 were maintained in McCoy's 5a medium (Invitrogen, Carlsbad, CA, USA), respectively supplemented with $10 \%$ fetal bovine serum (HyClone, Logan, UT, USA), penicillin and streptomycin (Pen-Strep; Invitrogen) at $37^{\circ} \mathrm{C}$ in a humidified atmosphere containing $5 \% \mathrm{CO}_{2}$.

Immunofluorescent staining. The $7 \mu \mathrm{m}$ thick frozen sections were obtained by using cryostat and fixed by $4 \%$ paraformaldehyde (Wako Pure Chemical Industries). After blocking, sections were incubated with rat anti-SSEA-3 monoclonal antibody (MAB4303, Millipore, Billerica, MA, USA) and Alexa Fluor 488 mouse anti-human E-cadherin (BD Pharmingen, San Diego, CA, USA). Alexa Fluor 594 goat anti-rat antibody (Invitrogen) was used as a secondary antibody. Rat IgM isotype (BD Pharmingen) was used as the control. Sections were mounted with ProLong Gold Antifade Reagent with DAPI (Invitrogen) and viewed with a fluorescent microscope (BZ-9000, Keyence, Osaka, Japan).

For immunocytochemistry, cells were cultured on 8-well culture slides (BD Falcon, Franklin Lakes, $\mathrm{NJ}$ ) at the concentration of 20,000 cells/well for $24 \mathrm{~h}$, fixed with $4 \%$ paraformaldehyde, then permealized with Triton X-100 (SigmaAldrich, St. Louis, MO, USA) and processed using the same protocol as the frozen sections.

Flow cytometry. Cells were dissociated with Accutase (Invitrogen), blocked with FcR blocking reagent (BD Biosciences) and incubated with antibodies as follows; antiSSEA-3 (MAB4303, Millipore), anti-CD105 (APC-conjugated,
BioLegend, San Diego, CA, USA), anti-rat IgM (APC-conjugated; Jackson ImmunoResearch Laboratories, West Grove, PA, FITC-conjugated; BD Biosciences, San Jose, CA, USA), anti-CD44, anti-CD24, anti-CD26 (conjugated form, BD Biosciences) and AldeFluor (Stemcell Technologies, BC, Canada). 7-AAD (BD Biosciences) was used to eliminate dead cells. Cells were analyzed and isolated by using FACSAriaII equipped with FACS Diva software (BD Biosciences).

$R N A$ preparation and quantitative real-time $P C R$. Total RNA was isolated using TRIzol reagent (Invitrogen). In all cases, $1 \mu \mathrm{g}$ of total RNA was reverse-transcribed with High Capacity RNA-to-cDNA Master Mix (Applied Biosystems, Foster, CA, USA) following the manufacturer's instructions. For quantitative assessments, real-time RT-PCR analysis was performed with the LightCycler TaqMan Master kit (Roche Diagnostics, Tokyo) and the LightCycler 480 system (Roche Applied Science, Indianapolis, IN, USA). The sequences of the primers used were as follows: GAPDH (NM_002046.3) sense primer 5'-AGCCACATCGCTCAGACAC-3' and antisense primer 5'-GCCCAATACGACCAAATCC-3'; OCT3/4 (NM_203289.3) sense primer 5'-AATCCAGTCCCAGGACATCA-3' and antisense primer 5'-TGGCTGAATACCTTCCCAAA-3'; NANOG (NM_024865.2) sense primer 5'-ATGCCTCACACGGAGAC TGT-3' and antisense primer 5'-AGGGCTGTCCTGAATAA GCA-3'; SOX2 (NM_003106.2) sense primer 5'-CTCCGGGA CATGATCAGC-3' and antisense primer 5'-CTGGGACAT GTGAAGTCTGC-3'; $c$-MYC (NM_002467.4) sense primer 5'-CACCAGCAGCGACTCTGA-3' and antisense primer 5'-GATCCAGACTCTGACCTTTTGC-3'.

Western blot analysis. Western blot analysis was performed as described previously (18). The following antibodies, at appropriate concentrations, were applied on membranes after the transfer of a sodium dodecyl sulfate-polyacrylamide gel (Bio-Rad Laboratories, Hercules, CA, USA): mouse anti-Oct4 monoclonal antibody (MAB4305, Millipore), anti-Nanog goat polyclonal antibody (R\&D Systems, Minneapolis, MN, USA), anti-Sox2 rabbit polyclonal antibody (MBL, Nagoya, Japan), anti-cMyc, anti-p21 ${ }^{\text {Cipl/Waf1 }}$, anti-p2 $7^{\text {Kip1 }}$ and anti-cyclin D1 mouse monoclonal antibodies, anti-cyclin A2 and anti-cyclin B1 rabbit monoclonal antibodies (Santa Cruz Biotechnology, Santa Cruz, CA, USA), anti-p16 mouse monoclonal antibody (BD Pharmingen), and anti-cyclin E mouse monoclonal antibody (Calbiochem, La Jolla, CA, USA). Equal loading of the protein samples was confirmed by parallel western blots for actin with anti-actin rabbit polyclonal antibody (SigmaAldrich).

Cell cycle analysis. To synchronize cell cycle, double-thymidine block (DTB) method $(19,20)$ was used. On $10 \mathrm{~cm}$ dishes, $1 \times 10^{6}$ cells of HCT116 were cultured in media containing $2.5 \mathrm{mM}$ thymidine (Sigma-Aldrich) for $18 \mathrm{~h}$, placed in thymidine-free media for $12 \mathrm{~h}$, and followed by an additional $18 \mathrm{~h}$ in thymidine containing media. Between the exchange of media, dishes were washed twice with PBS (Wako Pure Chemical Industries). Cell cycle was analyzed at 0, 4, 8, 16 and $24 \mathrm{~h}$ after release of block with PI/RNase Staining Buffer (BD Biosciences) on FACSAriaII and data analysis was performed using FlowJo software (Tree Star, Ashland, OR, USA). 
a
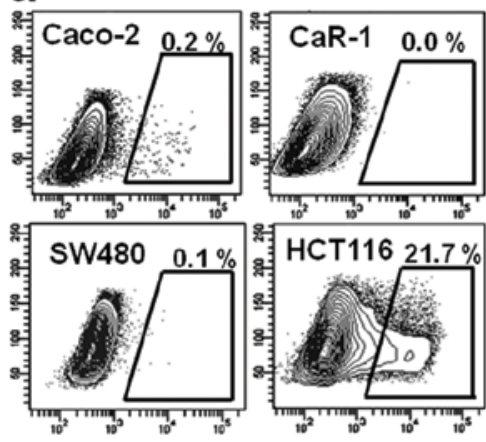

b

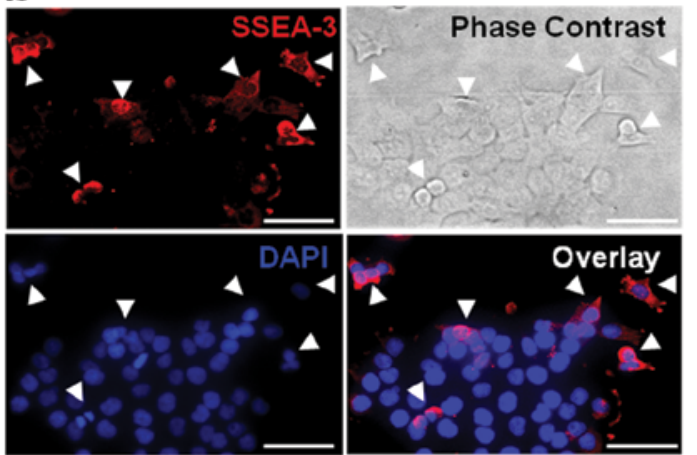

C
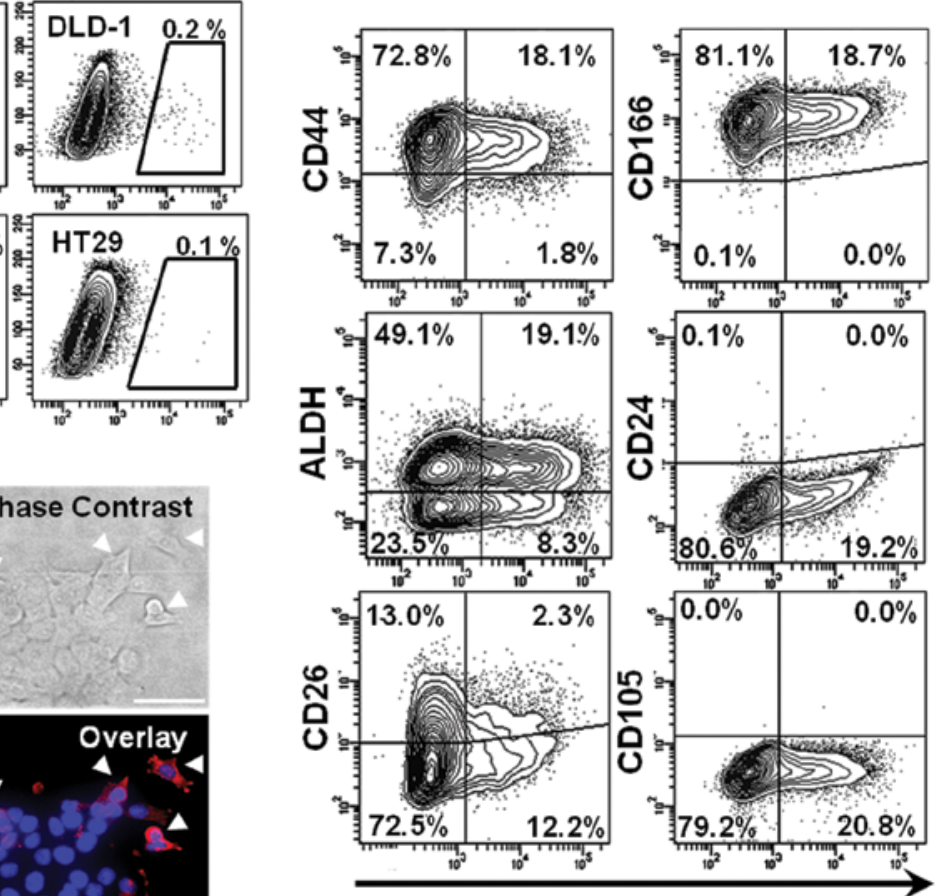

SSEA-3

Figure 1. Flow cytometry analysis of human CRC cell lines with an anti-SSEA-3 antibody and other stem cell markers, and immunofluorescent staining of a human CRC cell line. (a) Representative dot plots of human CRC cell lines incubated with an anti-SSEA-3 antibody. Boxes show SSEA-3 ${ }^{+}$cells, and the numbers above the boxes indicate the ratio of the SSEA- $3^{+}$population in each cell line. (b) Immunofluorescent staining of HCT116 cells with anti-SSEA-3 (red) and DAPI (blue), and phase-contrast image. Arrow heads indicate SSEA- $3^{+}$cells. Original magnification, x200. Scale bar, $50 \mu \mathrm{m}$. (c) Representative dot plots of HCT116 cells incubated with anti-SSEA-3 and anti-CD44, anti-CD166, anti-ALDH, anti-CD24, anti-CD26 and anti-CD105 antibodies. The numbers indicate the ratio of the population.

Sphere formation assay. Single cells of isolated SSEA-3+ and SSEA-3- in HCT116 were seeded in 96-well ultra-low attachment plates (Corning, New York, NY, USA), cultured in mTeSR1 (Stem Cell Technologies) media without serum. Sphere formation was assessed 28 days after seeding. In each of the 48 wells, the number of formed spheres was calculated. This study was performed as triplicated study.

Cell proliferation assay. Isolated HCT116 SSEA-3- and SSEA- $3^{+}$cells were put into 96 -well plates at a density of $2 \times 10^{3}$ cells in $100 \mu \mathrm{l}$ medium per well, cultured for $24 \mathrm{~h}$ in McCoy's 5A medium supplemented with $10 \%$ FBS, and then cultured for an additional 24, 48, 72 and $96 \mathrm{~h}$. Cell proliferation was assessed with Cell Counting kit- 8 incorporating WST-8 (Dojindo Molecular Technologies, Kumamoto, Japan) following the manufacturer's instructions with a plate reader (Model 680XR; Bio-Rad Laboratories).

Xenotransplantation. To assess tumorigenic properties, each of the 5,000 and 1,000 SSEA-3 ${ }^{-}$and SSEA-3 ${ }^{+}$cells were inoculated into dorsal flanks of 8-week-old NOD/SCID mice (CREA, Tokyo) with a mixture of Matrigel matrix (BD Biosciences). Tumor growth was monitored every three or four days by measurement with calipers and the volume of the subcutaneous tumor was calculated by the following formula; $v=l \times w^{2} / 2$, where $v=$ volume, $l=$ length and $w=$ width (21).
Statistical analysis. The relationships among gene expressions, cell counts, and tumor volume were analyzed using Student's t-tests. All tests were analyzed with JMP 9 software (SAS Institute, Cary, NC, USA). A value of $\mathrm{P}<0.05$ was taken as statistically significant.

\section{Results}

SSEA-3 expression in CRC cell lines. To assess SSEA-3 expression in $\mathrm{CRC}$, flow-cytometric analysis was performed on 6 CRC cell lines. It indicated that a small number of SSEA-3+ population existed in most of the CRC cell lines; 0.1 to $0.2 \%$ in Caco-2, DLD-1, HT-29 and SW480. Among those tested, HCT116 had the highest expression level; it had as much as $21.7 \pm 8.3 \%$ SSEA- $3^{+}$population. In CaR-1 cells, expression of SSEA-3 could not be identified in this triplicate study (Fig. 1a). To confirm SSEA-3 expression in HCT116 cells, immunofluorescent staining of SSEA-3 was performed. Consistent with the results of flow cytometric analysis, a small number of HCT116 cells expressed SSEA-3 (Fig. 1b). In this study, HCT116 was used for the following analyses because it had abundant SSEA- $3^{+}$cells and it was easy to isolate SSEA-3 ${ }^{-}$and SSEA-3 ${ }^{+}$ populations.

Correlation of SSEA-3 and stem cell markers. To clarify the correlation between SSEA-3 and representative colorectal 


\section{a}

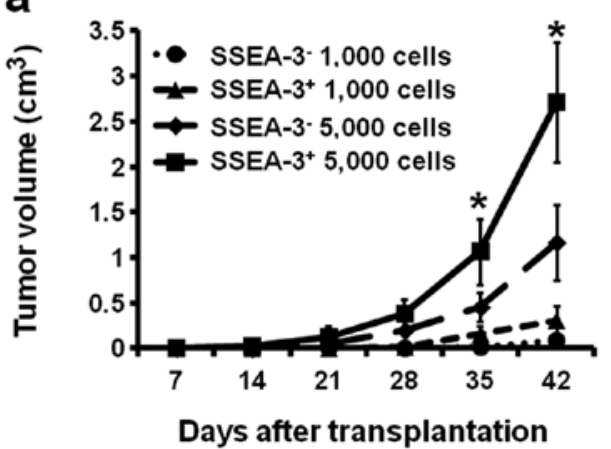

C

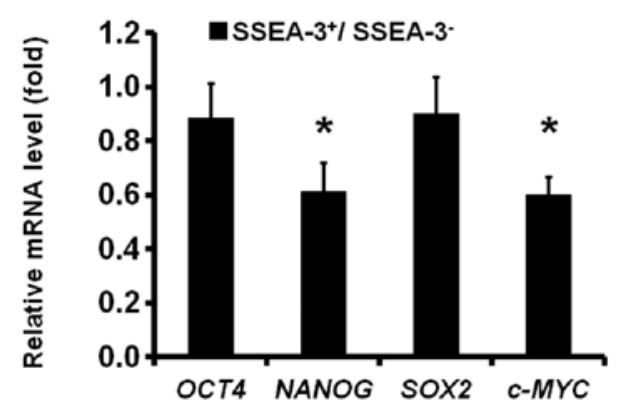

b

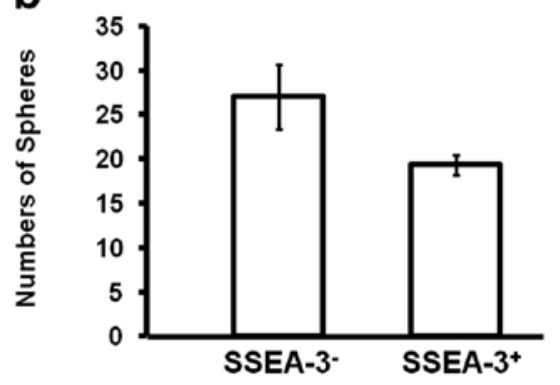

d

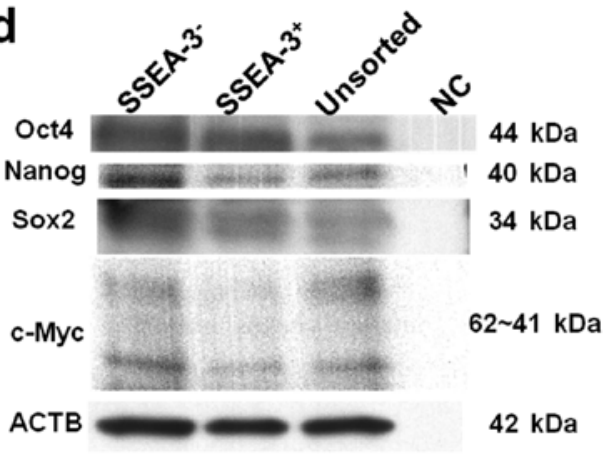

Figure 2. Tumorigenicity assay, sphere formation assay and qRT-PCR and western blot analyses of pluripotency-associated genes in flow cytometry-isolated SSEA-3 ${ }^{-}$and SSEA- $3^{+}$HCT116 cells. (a) Xenotransplant results of flow cytometry-isolated 1,000 and 5,000 SSEA-3 and SSEA-3 ${ }^{+}$HCT116 cells. (b) Sphere formation results of flow cytometry-isolated SSEA-3 and SSEA-3 ${ }^{+}$HCT116 cells. Sphere numbers formed in 48 wells 28 days after seed are shown. (c) Expression of pluripotency-associated genes (OCT4, NANOG, SOX2 and c-MYC) evaluated by the ratio normalized to GAPDH expression in SSEA- $3^{+}$HCT116 cells relative to SSEA ${ }^{-}$cells as determined by qRT-PCR. (d) Expression of pluripotency-associated proteins of SSEA-3, SSEA-3 ${ }^{+}$and unsorted HCT116 cells. The asterisk indicates statistical significance.

CSC markers, HCT116 cells were double-stained with CD44, CD166, ALDH, CD24 and CD26 (22). In CD44+ fraction $19.9 \%$ of cells were SSEA-3 positive. In $\mathrm{CD} 166^{+}$fraction $18.7 \%$, in $\mathrm{ALDH}^{+}$fraction $28.0 \%$, in $\mathrm{CD} 24^{+}$fraction $0.0 \%$ and in $\mathrm{CD} 26^{+}$ fraction $15.0 \%$ of cells were SSEA-3 positive. These findings indicated that there was no apparent correlation between the representative CSC markers and SSEA-3 expression (Fig. 1c). Next, correlation between the expression of SSEA-3 and CD105 [a specific mesenchymal/Muse cell marker (23)] was assessed. In contrast to the abundant expression of SSEA-3, expression of CD105 could not be identified in HCT116 cells, implying that properties of SSEA- $3^{+}$cancer cells may be somewhat different from those of Muse cells (Fig. 1c).

Correlation of SSEA-3 and tumorigenicity and sphere formation ability. To confirm if SSEA-3 expression status actually does not correlate with CSC characteristics, the tumorigenic activity of SSEA-3- and SSEA- $3^{+}$cells were assessed. The freshly isolated 1,000 and 5,000 SSEA- $3^{-}$and SSEA- $3^{+}$HCT116 cells were transplanted subcutaneously into NOD/SCID mice. Notably, though SSEA-3 expression was not correlated with representative colorectal CSC markers, SSEA- $3^{+}$cells revealed higher tumorigenic activity compared to that of SSEA-3- cells in both 1,000 and 5,000 cell inoculations (Fig. 2a). To assess self-renewal ability, sphere formation assays were performed three times. This revealed that SSEA- $3^{+}$cells actually did form spheres, but the number of formed spheres was significantly smaller $(\mathrm{P}<0.05)$ compared to that of SSEA-3- cells (Fig. 2b).
Correlation of SSEA-3 and pluripotency-associated genes. To resolve the reason why SSEA- $3^{+}$cells reveal high tumorigenic activity, expressions of pluripotency-associated genes; OCT4, $N A N O G, S O X 2$ and $c-M Y C$, which play key roles in iPS cell induction (10) and are overexpressed in Muse cells (23), were analyzed on freshly isolated SSEA-3 ${ }^{-}$and SSEA-3 ${ }^{+}$HCT116 cells by qRT-PCR. The results indicated that the expression of $N A N O G$ and $c-M Y C$ in SSEA- $3^{+}$cell population was significantly lower than that of SSEA-3-, while the expression of OCT4 and SOX2 were not significantly different when stratified by SSEA-3 levels (Fig. 2c), both of which were consistent with the results from the western blot analysis (Fig. 2d). These results indicated that SSEA- $3^{+}$cancer cells had clearly distinct properties from Muse cells (23).

Correlation of SSEA-3 and proliferation ability and cell cycle. To elucidate the background of the difference of tumorigenic ability, cell proliferation ability of SSEA-3 ${ }^{-}$and SSEA-3 ${ }^{+}$ cells were assessed. As compared to SSEA-3- cells, SSEA-3 ${ }^{+}$ cells showed significantly higher proliferative activity in vitro $(\mathrm{P}<0.05$ at 3, 4 and 5 days after seeding) (Fig. 3a). To clarify the background of high proliferation ability of SSEA- $3^{+}$cells observed in vivo and in vitro, the cell cycle status of SSEA-3 and SSEA- $3^{+}$cells were assessed, because SSEA- $3^{+}$cells in ES cells were reported to show rapid cell cycles (24). Cell cycle analysis using HCT116 cells synchronized with a doublethymidine block method revealed faster entry from G1 to $\mathrm{S}$ and from $\mathrm{S}$ to $\mathrm{G} 2 / \mathrm{M}$ phase in SSEA-3 ${ }^{+}$cells (Fig. $2 \mathrm{c}$ ); there were 


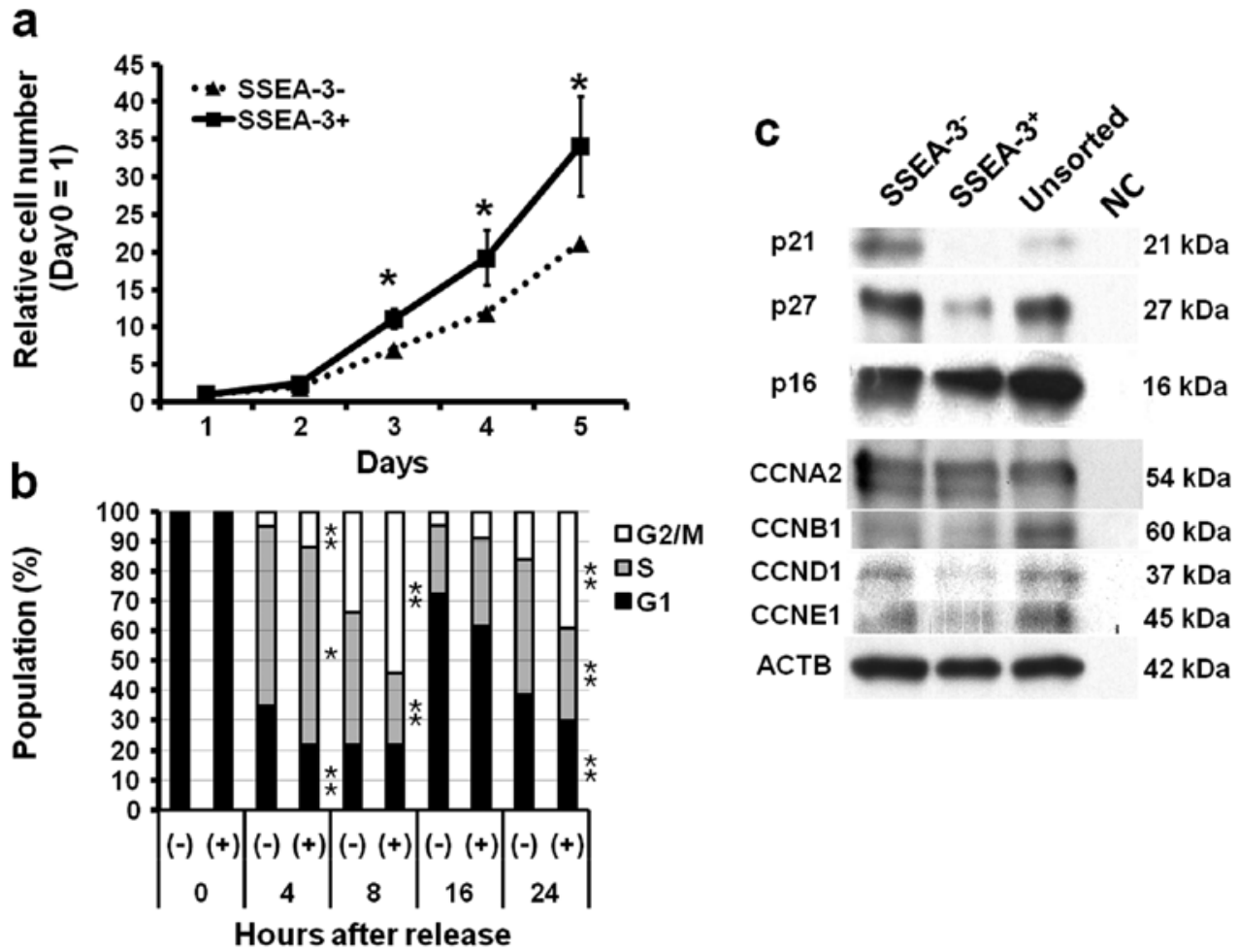

Figure 3. Proliferation assay, cell cycle analysis and western blot analyses of cell cycle related factors. (a) Proliferation assay of isolated SSEA-3 ${ }^{-}$and SSEA- $3^{+}$ HCT116 cells. Cell numbers relative to those on day 1 from day 2 to 5 are shown. (b) Cell cycle analysis of flow cytometry-isolated SSEA-3 and SSEA- $3^{+}$ HCT116 after synchronization with DTB method. Significant differences ( $\mathrm{P}<0.05$ and $\left.{ }^{* *} \mathrm{P}<0.01\right)$ were obtained from three independent experiments. (c) Western blot analysis of protein from non-synchronized SSEA-3 ${ }^{-}$and SSEA- $3^{+}$HCT116 cell lysate on representative cyclins and cyclin-dependent kinase inhibitors.

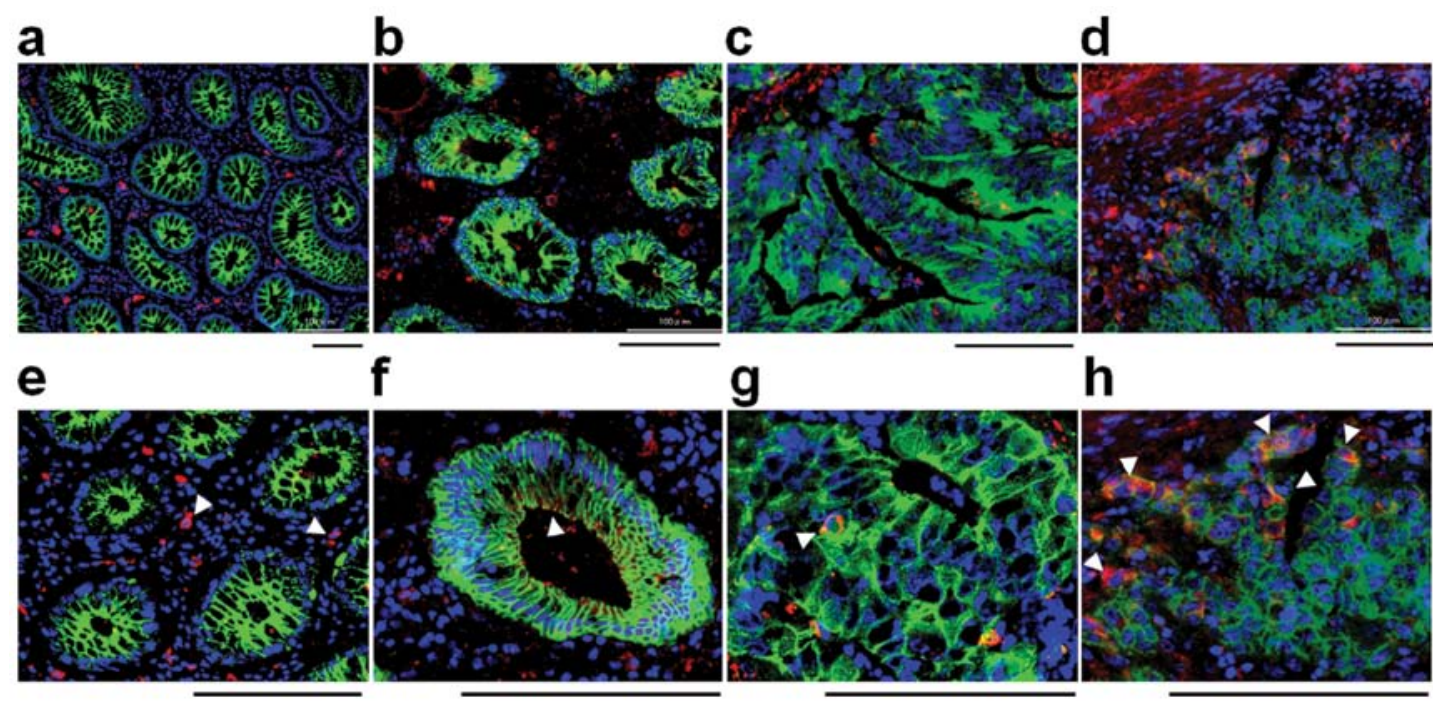

Figure 4. Immunofluorescent staining of clinical samples with anti-SSEA-3 and anti-E-cadherin antibodies. Merged images of normal epithelium (a and e), well differentiated ( $b$ and f), moderately differentiated ( $\mathrm{c}$ and $\mathrm{g}$ ) and poorly differentiated adenocarcinoma $(\mathrm{d}$ and $\mathrm{h}$ ), stained with anti-SSEA-3 (red), anti-E-cadherin (green) and DAPI (blue). The lower images (e-h) depict higher magnification of typically stained patterns. Arrowheads indicate SSEA-3 $3^{+}$cells. Scale bar, $100 \mu \mathrm{m}$.

significant increases of $\mathrm{S}$ phase $(\mathrm{P}<0.05$ at $4 \mathrm{~h})$ and $\mathrm{G} 2 / \mathrm{M}$ phase $(\mathrm{P}<0.05$ at 4,8 , and $24 \mathrm{~h})$ population and significant decrease of G1 phase $(\mathrm{P}<0.05$ at 4 and $24 \mathrm{~h})$ and $\mathrm{S}$ phase $(\mathrm{P}<0.05$ at 8 and $24 \mathrm{~h}$ ) population in SSEA-3+ cells as compared to SSEA-3 cells (Fig. 3b). Western blotting showed the decrease of p21 Cip1/Waf 1 and $\mathrm{p} 27^{\mathrm{Kip} 1}$ (hereafter designated p21 and p27, respectively) in
SSEA- $3^{+}$cells, which are consistent with the results of cell cycle analyses, while cyclins showed no apparent difference between SSEA-3 ${ }^{-}$and SSEA-3 ${ }^{+}$cells (Fig. $3 \mathrm{c}$ ).

SSEA-3 expression in colorectal normal and cancer tissues. To investigate SSEA-3 expression in normal colorectal epithelia 
and colorectal cancer, frozen specimens, which contain both normal and cancerous areas in the same section were stained with SSEA-3 (2 cases of well differentiated, 6 cases of moderately differentiated and 2 cases of poorly differentiated adenocarcinoma). In normal areas, small numbers of stromal cells were strongly positive for SSEA-3, but epithelial cells were not apparently positive for SSEA-3 (Fig. 4a). In cancer areas, SSEA- $3^{+}$cancer cells were identified in $50 \%$ ( 1 out of 2 cases) of well differentiated, in $66.7 \%$ (4 out of 6 cases) of moderately differentiated and in $100 \%$ (2 out of 2 ) of poorly differentiated adenocarcinomas (Fig. 4b-d). The positive ratio and the expression level of SSEA-3 had a tendency to be high in poorly differentiated adenocarcinomas but low in those that were well differentiated (Fig. 4d).

\section{Discussion}

In this study, we directly revealed the existence of SSEA-3 ${ }^{+}$ population in gastroenterological cancer, as far as we know, for the first time. We have also clarified the cellular properties of SSEA- $3^{+}$cells; highly tumorigenic and high proliferative ability.

Though we could not identify a significant correlation between SSEA-3 expression and representative colorectal CSC markers (CD44, CD166, ALDH, CD24 and CD26), we revealed that SSEA-3 ${ }^{+}$cells in the HCT116 CRC cell line possess high tumorigenic ability, which is one of the representative properties of CSCs, in immunodeficient mice. In sphere formation assay, SSEA- $3^{+}$cells showed sufficient potency of sphere formation ability, but it was significantly lower than that of SSEA-3- cells. These results indicate that SSEA- $3^{+}$cells in CRC retain immature phenotypes, but have diminished stem cell properties, including self-renewal capacity and pluripotency. In the CRC cell line, we have identified abundant SSEA- $3^{+}$expression in HCT116 cells which possess unique properties; they do not differentiate, even with forced differentiation process and contain mainly CSCs (25). This report also supports our findings that SSEA- $3^{+}$cells in CRC possess immature phenotypes. A recent study indicated that colorectal CSCs involve at least three characteristic sub-populations; long-term tumor initiating cells (LT-TIC), tumor transient amplifying cells (T-TAC) and delayed contributing TIC (DC-TIC) (26). On this basis, we hypothesized that SSEA- $3^{+}$cells play a role as T-TAC in cancer progression. To resolve these questions, we assessed the proliferation activity and cell cycle status in SSEA- $3^{+}$cells, the data indicated that SSEA $-3^{+}$cells had high proliferative activity. In the study of ES cells, SSEA- $3^{+}$cells reportedly showed a faster cell cycle (24), and we further detected the decreased expression of cyclin-dependent kinase inhibitors p21 and p27 in SSEA-3 ${ }^{+}$ cells. p27 is not only a cell cycle inhibitory factor of G1/S transition, but also a differentiation-promoting factor (27), and decreased expression of $\mathrm{p} 27$ in SSEA- $3^{+}$population has been reported in teratocarcinoma (28). p21 negatively regulates not only G1/S transition, but also G2/M transition (29), and its relation to SSEA-3 expression has not been reported. These findings also supported our hypothesis.

SSEA-3 is known as a specific marker for Muse cells, and Muse cells co-express CD105 (23). In this study, we could not identify CD105 expression in SSEA-3 expressing CRC cells, which indicates that SSEA- $3^{+}$cells in CRC differ from Muse cells in marker expression. In addition, though Muse cells overexpress iPS related genes (Oct3/4, NANOG, SOX2 and c-Myc) (23), the expression pattern of iPS-related genes in SSEA- $3^{+}$CRC cells was clearly different from that of Muse cells, suggesting that cellular characteristics of SSEA- $3^{+}$cells in $\mathrm{CRC}$ are also different from Muse cells. Moreover, the immunofluorescent finding that no SSEA- $3^{+}$cells were detected in normal colorectal epithelia also supports our findings.

In this study, unfortunately, we could not show a correlation between SSEA-3 and clinical outcome because immunohistochemical analysis for SSEA-3 on formalin-fixed paraffin embedded (FFPE) samples was very difficult. SSEA-3 is a glycolipid and can be lost in formalin, ethanol or methanol, meaning that FFPE samples are not accurate. Though Chang et al showed the existence of SSEA- $3^{+}$cells in normal human colorectal tissues using FFPE samples (17), we could not obtain reproducible immunohistochemical staining of SSEA-3 in FFPE sections. For the same reason, qRT-PCR analysis and western blot analysis are not routine methodologies for SSEA-3. For further studies, it is necessary to identify a novel marker that co-expresses on SSEA- $3^{+}$cells in CRC cells.

In the study of CSCs, especially in solid tumors, tumorigenic properties have been considered to reveal CSC properties. Based on this study, we imply that the assessment of tumorigenicity is not sufficient to isolate CSC subsets, LT-TIC, T-TAC and DC-TIC. To isolate and identify CSC subsets, it may be essential to establish a new approach with a combination of repeated serial transplantation assays.

\section{Acknowledgements}

We thank Dr Mari Dezawa and Dr Masaaki Kitada at Tohoku University and Dr Eiichi Morii at Osaka University for a valuable discussion on immunohistochemical sections. This study was supported by a Grant-in-Aid for Cancer Research from the Ministry of Education, Science, Sports and Culture Technology, Japan, to H.Y. (grant no. 21390360).

\section{References}

1. Ben-Porath I, Thomson MW, Carey VJ, et al: An embryonic stem cell-like gene expression signature in poorly differentiated aggressive human tumors. Nat Genet 40: 499-507, 2008.

2. Levina V, Marrangoni AM, DeMarco R, Gorelik E and Lokshin AE: Drug-selected human lung cancer stem cells: cytokine network, tumorigenic and metastatic properties. PLoS One 3: E3077, 2008.

3. Rajasekhar VK, Studer L, Gerald W, Socci ND and Scher HI: Tumour-initiating stem-like cells in human prostate cancer exhibit increased NF-kappaB signalling. Nat Commun 2: 162, 2011.

4. Wright AJ and Andrews PW: Surface marker antigens in the characterization of human embryonic stem cells. Stem Cell Res 3: 3-11, 2009.

5. Solter D and Knowles BB: Monoclonal antibody defining a stagespecific mouse embryonic antigen (SSEA-1). Proc Natl Acad Sci USA 75: 5565-5569, 1978.

6. Shevinsky LH, Knowles BB, Damjanov I and SolterD: Monoclonal antibody to murine embryos defines a stage-specific embryonic antigen expressed on mouse embryos and human teratocarcinoma cells. Cell 30: 697-705, 1982.

7. Kannagi R, Cochran NA, Ishigami F, et al: Stage-specific embryonic antigens (SSEA-3 and -4) are epitopes of a unique globo-series ganglioside isolated from human teratocarcinoma cells. EMBO J 2: 2355-2361, 1983.

8. Solter D, Shevinsky L, Knowles BB and Strickland S: The induction of antigenic changes in a teratocarcinoma stem cell line (F9) by retinoic acid. Dev Biol 70: 515-521, 1979. 
9. Evans MJ and Kaufman MH: Establishment in culture of pluripotential cells from mouse embryos. Nature 292: 154-156, 1981.

10. Takahashi $\mathrm{K}$ and Yamanaka S: Induction of pluripotent stem cells from mouse embryonic and adult fibroblast cultures by defined factors. Cell 126: 663-676, 2006.

11. Thomson JA, Itskovitz-Eldor J, Shapiro SS, et al: Embryonic stem cell lines derived from human blastocysts. Science 282: 1145-1147, 1998.

12. Takahashi K, Tanabe K, Ohnuki M, et al: Induction of pluripotent stem cells from adult human fibroblasts by defined factors. Cell 131: 861-872, 2007.

13. Kuroda Y, Kitada M, Wakao S, et al: Unique multipotent cells in adult human mesenchymal cell populations. Proc Natl Acad Sci USA 107: 8639-8643, 2010.

14. Kannagi R: Carbohydrate-mediated cell adhesion involved in hematogenous metastasis of cancer. Glycoconj J 14: 577-584, 1997.

15. Son MJ, Woolard K, Nam DH, Lee J and Fine HA: SSEA-1 is an enrichment marker for tumor-initiating cells in human glioblastoma. Cell Stem Cell 4: 440-452, 2009.

16. Ye F, Li Y, Hu Y, Zhou C and Chen H: Stage-specific embryonic antigen 4 expression in epithelial ovarian carcinoma. Int $\mathrm{J}$ Gynecol Cancer 20: 958-964, 2010.

17. Chang WW, Lee $\mathrm{CH}$, Lee $\mathrm{P}$, et al: Expression of Globo $\mathrm{H}$ and SSEA 3 in breast cancer stem cells and the involvement of fucosyl transferases 1 and 2 in Globo H synthesis. Proc Natl Acad Sci USA 105: 11667-11672, 2008.

18. Yamamoto H, Kondo M, Nakamori S, et al: JTE-522, a cyclooxygenase-2 inhibitor, is an effective chemopreventive agent against rat experimental liver fibrosis1. Gastroenterology 125: 556-571, 2003.

19. Bello LJ: Studies on gene activity in synchronized culture of mammalian cells. Biochim Biophys Acta 179: 204-213, 1969.

20. Wilker EW, van Vugt MA, Artim SA, et al: 14-3-3sigma controls mitotic translation to facilitate cytokinesis. Nature 446: 329-332, 2007.
21. Ware JL and DeLong ER: Influence of tumour size on human prostate tumour metastasis in athymic nude mice. Br J Cancer 51: 419-423, 1985.

22. Todaro M, Francipane MG, Medema JP and Stassi G: Colon cancer stem cells: promise of targeted therapy. Gastroenterology 138: 2151-2162, 2010

23. Wakao S, Kitada M, Kuroda Y, et al: Multilineage-differentiating stress-enduring (Muse) cells are a primary source of induced pluripotent stem cells in human fibroblasts. Proc Natl Acad Sci USA 108: 9875-9880, 2011.

24. Stewart MH, Bosse M, Chadwick K, Menendez P, Bendall SC and Bhatia M: Clonal isolation of hESCs reveals heterogeneity within the pluripotent stem cell compartment. Nat Methods 3: 807-815, 2006

25. Yeung TM, Gandhi SC, Wilding JL, Muschel R and Bodmer WF: Cancer stem cells from colorectal cancer-derived cell lines. Proc Natl Acad Sci USA 107: 3722-3727, 2010.

26. Dieter SM, Ball CR, Hoffmann CM, et al: Distinct types of tumor-initiating cells form human colon cancer tumors and metastases. Cell Stem Cell 9: 357-365, 2011.

27. Wander SA, Zhao D and Slingerland JM: p27: a barometer of signaling deregulation and potential predictor of response to targeted therapies. Clin Cancer Res 17: 12-18, 2011.

28. Baldassarre G, Barone MV, Belletti B, et al: Key role of the cyclin-dependent kinase inhibitor p27kip1 for embryonal carcinoma cell survival and differentiation. Oncogene 18: 6241-6251, 1999

29. Niculescu AB III, Chen X, Smeets M, Hengst L, Prives C and Reed SI: Effects of p21(Cip1/Waf1) at both the G1/S and the G2/M cell cycle transitions: $\mathrm{pRb}$ is a critical determinant in blocking DNA replication and in preventing endoreduplication. Mol Cell Biol 18: 629-643, 1998. 